\title{
Symptom variability in patients with severe COPD: a pan-European cross- sectional study
}

\author{
R. Kessler*, M.R. Partridge ${ }^{\#, ~ M . ~ M i r a v i t l l e s ", ~ M . ~ C a z z o l a ~}{ }^{+, \S}$, \\ C. Vogelmeier ${ }^{f}$, D. Leynaud ${ }^{* *}$ and J. Ostinelli**
}

ABSTRACT: In between exacerbations, chronic obstructive pulmonary disease (COPD) is usually regarded as a stable condition, but there is increasing recognition of variability in this state. This cross-sectional study assessed patients' perception of symptom variability.

Participants were outpatients $>\mathbf{4 5}$ yrs old with COPD, current or ex-smokers, forced expiratory volume in $1 \mathrm{~s}\left(F V_{1}\right)<50 \%$ predicted, FEV 1 forced vital capacity $<0.7$ and no exacerbation leading to therapeutic intervention in the previous 3 months. Patients' perceptions of COPD symptoms and their impact on daily life activities were recorded. Alterations in therapy use in response to COPD worsening were also recorded.

COPD symptoms were experienced by 2,258 (92.5\%) out of 2,441 patients during the 7 days before interview. Breathlessness was the most common symptom (72.5\%). Daily and/or weekly symptom variability was reported by $62.7 \%$ of symptomatic patients; the morning was the worst time of day. Factors associated with perception of variability of breathlessness included younger age, symptom severity and recruitment to the study by general practitioners. The perception of variability was significantly different between European countries or regions.

Patient-perceived COPD symptoms vary over the day and the week, and impact on daily activities; morning being the worst time of day. The majority of patients appear not to adjust treatment when symptoms worsen.

KEYWORDS: Breathlessness, chronic obstructive pulmonary disease, symptoms, symptom variability, treatment

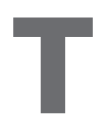
he most common symptoms of chronic obstructive pulmonary disease (COPD) are chronic cough, breathlessness, sputum production, wheezing and chest tightness [1].

Circadian variation of symptoms and lung function is a well known feature of asthma [2-4]. Circadian variations in lung function have also been described in patients with stable COPD, but have received considerably less attention [5-8]. COPD symptoms such as breathlessness are typically described as "characteristically persistent and progressive" [1]. Patients report that they are most fearful of COPD exacerbations and needing care from others. However, in between exacerbations they also experience "good days and bad days" [1,5]. Several studies have been undertaken to quantify the impact of the disease on daily life and well-being according to symptoms, severity, exacerbation state, emotional state or patients' perceptions of their physical function [5, 9-19]. Even though these studies have described fluctuations of COPD symptoms in individuals over a day, from day to day, and over longer periods of time [20-22], no published study has investigated symptom variability within a large group of COPD patients. This study describes patient perceptions of COPD symptom variability throughout the day, week and year in a large cohort of outpatients.

\section{METHODS}

\section{Patients}

This pan-European, cross-sectional, observational study was conducted in 17 countries: Austria, Belgium, Denmark, Finland, France, Germany, Greece, Ireland, Italy, the Netherlands, Norway, Portugal, Spain, Sweden, Switzerland, Turkey and the UK (NCT00722267). The study was
AFFILIATIONS

*Dept of Pneumology, Nouvel Hôpital Civil, Strasbourg, **Medical Dept, AstraZeneca, RueilMalmaison, France.

\#Imperial College London, NHLI Division, Charing Cross Hospital, London, UK

"Fundacio Clinic, IDIBAPS, Ciber de Enfermedades Respiratorias (CIBERES), Hospital Clinic, Barcelona, Spain.

${ }^{+}$Dept of Internal Medicine, University of Rome "Tor Vergata",

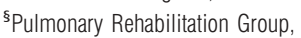
San Raffaele Pisana Hospital, Rome, Italy.

${ }^{f}$ Division for Respiratory Diseases, University of Marburg, Marburg, Germany.

CORRESPONDENCE

R. Kessler

Dept of Pneumology

Nouvel Hôpital Civil

1 Place de l'Hôpital

Strasbourg 67000

France

E-mail: Romain.Kessler@chru-

strasbourg.fr

Received:

March 312010

Accepted after revision:

Oct 132010

First published online:

Nov 292010 
conducted in accordance with the Declaration of Helsinki and approved by ethics committees (local and/or national) at each of the participating centres, according to local regulations. All patients provided informed consent to participate.

For inclusion in the study, subjects must have fulfilled all of the following criteria: outpatients over 45 yrs old, a COPD diagnosis, current or ex-smoker for $\geqslant 10$ pack-yrs, forced expiratory volume in $1 \mathrm{~s}(\mathrm{FEV} 1)<50 \%$ predicted and FEV1/forced vital capacity (FVC) $<0.7$ (pre-bronchodilator).

Patients with an ongoing COPD exacerbation or who had experienced a severe exacerbation, defined by a worsening of COPD symptoms leading to treatment with antibiotics and/or a short course of oral steroids and/or hospitalisation or emergency room visit, within the previous 3 months, were excluded. Other exclusion criteria were: history of asthma or allergic rhinitis, lung cancer or any other significant respiratory disease, such as bronchiectasis, lung fibrosis, interstitial lung disease, tuberculosis, or sarcoidosis; current participation in a clinical trial; an inability to understand the study procedures; or an inability or reluctance to answer a questionnaire over the telephone.

\section{Data collection}

Physicians (general practitioners (GPs) and respiratory physicians) were randomly selected from extensive lists of practitioners and contacted to confirm their willingness to participate. Selected physicians were required to manage a reasonable number of patients with severe COPD to enable them to recruit the expected number of patients in a maximum of 3 months, and be able to provide patient spirometric data (FEV1 and FEV1/FVC).

Physicians completed a case report form (CRF) for each patient, recording demographic information, time since COPD diagnosis, medical history, Medical Research Council (MRC) dyspnoea scores, smoking status, lung function measurements, and the use of current COPD medication and other concomitant medication.

In addition, patients were interviewed over the telephone by a trained researcher, independent from the physician and the study sponsor. The interview consisted of a questionnaire of 20-30 min duration, asking for the patient's reports of COPD symptoms and variability over the day, week and year; sleep quality, tiredness, depression and anxiety; the impact of symptoms on morning and other daily activities and the requirement for help to perform these activities; and alterations in the use of maintenance and rescue therapy in response to COPD worsening.

The patient questionnaire was translated into the languages appropriate for each country. To avoid differences in interpretation and to check the understanding of questions, a feasibility study was performed prior to patient recruitment. 15 stable COPD patients from different countries (France, Germany, Italy, Spain and the UK) were interviewed and minor changes were made to enhance the questionnaire for use in the present study.

Further information regarding details of the patient questionnaire, study validation and quality control are available in the online supplementary material.

\section{Data analysis}

Statistical analyses were conducted using SAS software version 8.2 (SAS Institute, Cary, NC, USA). The significance level was set at 5\%. Logistic regression analysis (PROC LOGISTIC in $S A S_{\mathbb{R}}$ ) was used to investigate the relationship between patient characteristics and the breathlessness variability-dependent variable. Only those patients who reported both breathlessness in the previous 7 days ("a little", "moderately", "very" or "extremely") and who answered the specific question regarding weekly or daily variability were included in the analysis. The dependent variable was "yes/no", with "yes" including patients who answered "yes, it varied a little" and "yes, it varied a lot" and "no" including patients who answered "no, it was about the same". As a preliminary analysis, all the parameters recorded were described according to the dependent variable (e.g. specific responses to questions in the patient questionnaire). A univariate analysis of individual dependent variables was performed to identify factors significantly associated with variability of breathlessness, the most troublesome symptom for COPD patients [1]. All factors with a p-value $<0.20$ in the univariate analysis were then included in a logistic regression model. In order to select the most relevant model, a backward procedure was used. All nonsignificant variables were removed one by one until all parameters reached a level of significance $\leqslant 0.05$. Interactions between the independent variables were not considered. Odds ratios are presented together with their associated 95\% confidence intervals.

As planned in the protocol, country- or region-specific statistical analyses were performed for countries in which the number of patients was equal to or exceeded the number of patients in Scandinavia (the region with the smallest population); this included France, Spain, Germany and the Scandinavian countries combined (Denmark, Finland, Sweden and Norway).

\section{RESULTS}

\section{Patient characteristics}

Of 3,642 patients initially recruited by 802 physicians $(40.9 \%$ GPs and $59.1 \%$ respiratory physicians), 2,441 patients had both telephone interview and lung function data (FEV1 and FEV1/ FVC) available in the CRF, and were therefore eligible for analysis (fig. 1). Patient characteristics are displayed in table 1.

In the 12 months preceding study enrolment, $61.6 \%(n=1,478)$ of patients had experienced at least one exacerbation. As a consequence, 492 patients (20.9\%) required hospitalisation, 459 patients $(19.4 \%)$ required a visit to the emergency room, and antibiotics or steroids were administered to 1,332 patients $(55.8 \%)$.

Of the total cohort of patients, 2,431 had available information on COPD medication; among them 2,402 (98.8\%) patients had been prescribed at least one COPD medication. The majority of patients were treated with inhaled corticosteroids associated with long-acting bronchodilators (table 1), and 12.4\% $(n=303)$ had enrolled in a rehabilitation programme. For prescribed rescue medication, $42.6 \%$ of patients were using short-acting $\beta_{2}$-agonists, $13.8 \%$ short-acting anticholinergics and $8.9 \%$ a combination of both.

Comorbidities were reported for $76.2 \%$ of patients $(n=1,861)$, with a significant proportion $(44.9 \% ; n=1,095)$ reporting more than one comorbid condition. As shown in table 2, hypertension was the most frequent comorbidity, followed by 


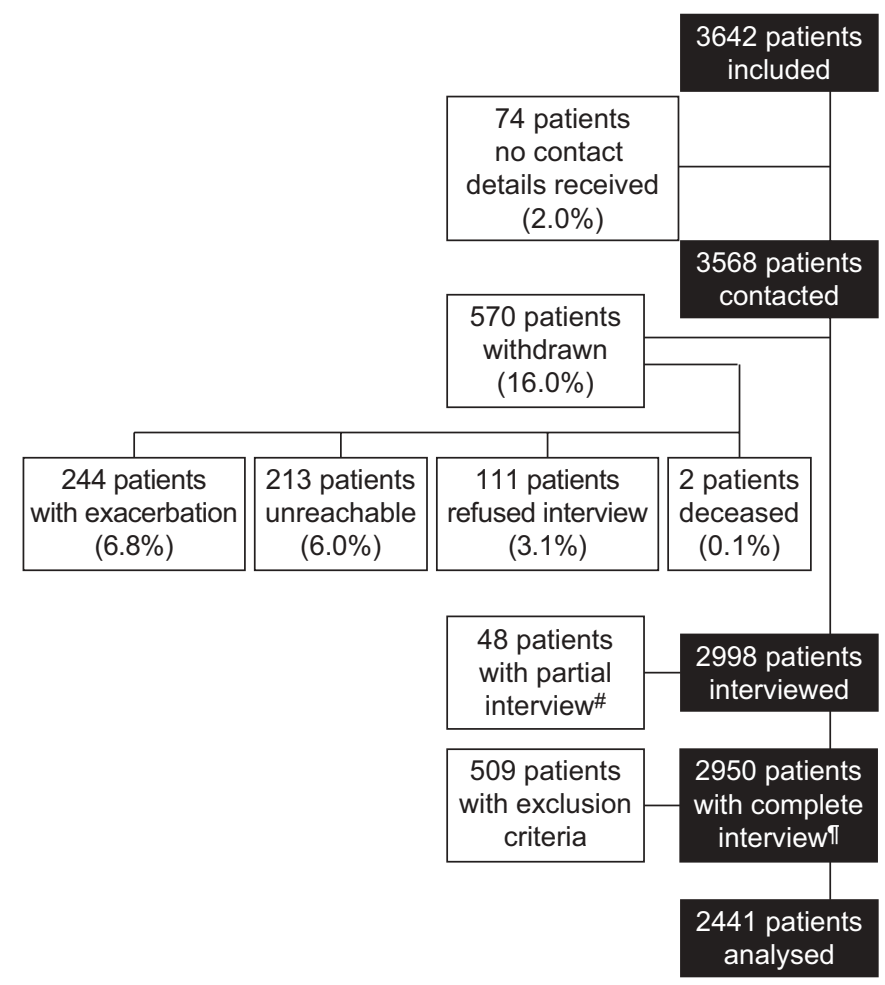

FIGURE 1. Patient recruitment flow chart. *: part $A$ of the questionnaire (symptoms) not completed; $\uparrow$ : part $\mathrm{A}$ of the questionnaire (symptoms) completed.

cardiovascular-related comorbidities (patients suffering from ischaemic heart disease and/or left heart failure), recorded for 478 patients $(19.6 \%)$. Concomitant medications were received by $60.9 \%$ of patients $(n=1,486$; table 2$)$.

\section{Symptom variability}

A total of $92.5 \%$ of patients reported experiencing at least one of the following symptoms in the 7 days prior to telephone interview: breathlessness, phlegm, cough, wheezing or chest tightness. As shown in figure 2, breathlessness was the most frequent symptom among the study participants $(72.5 \%)$. Among patients who reported breathlessness, a greater number reported tiredness due to their chest condition compared with patients who reported no respiratory symptoms $(70.4 \%$ versus $25.1 \%$ ). When asked how many days of the previous week their symptoms affected them the most, for every symptom, the two most common responses were 1-3 days (24.2-42.5\%, depending on the symptom) or every day (33.6-50.1\%, depending on the symptom). Patients who reported experiencing symptoms were also asked whether they perceived any variation of the symptoms over the course of a year, a week or a single day. Among symptomatic patients, $62.7 \%(n=1,403)$ perceived variability in one or more symptoms; $44.7 \%(n=975)$ throughout the day and $54.4 \%(n=1,211)$ throughout the week, respectively. Seasonal variation in respiratory symptoms were perceived by $59.5 \%(n=1,452)$ of patients; for those patients with available data, $55.9 \%(n=588)$ reported that their symptoms were at their worst during the winter.

Chest tightness was reported as the most variable symptom over the course of a week, followed by breathlessness,

\section{TABLE 1 Patient characteristics}

\begin{tabular}{|c|c|c|}
\hline & $\begin{array}{c}\text { Available } \\
\text { data }^{\#}\end{array}$ & \\
\hline Sex & $2440(99.96)$ & \\
\hline Male & & $1916(78.5)$ \\
\hline Female & & $524(21.5)$ \\
\hline Age yrs & $2427(99.43)$ & $67.3 \pm 9.4$ \\
\hline Body mass index $\mathrm{kg} \cdot \mathrm{m}^{-2}$ & $2394(98.07)$ & $26.0 \pm 5.0$ \\
\hline Smoking status & $2337(95.74)$ & \\
\hline Current smoker & & $565(24.2)$ \\
\hline Ex-smoker & & $1772(75.8)$ \\
\hline Time since COPD diagnosis yrs & $2365(96.89)$ & $8.9 \pm 7.6$ \\
\hline MRC dyspnoea score & $2429(99.51)$ & \\
\hline 1 & & $172(7.1)$ \\
\hline 2 & & 791 (32.6) \\
\hline 3 & & $803(33.1)$ \\
\hline 4 & & $561(23.1)$ \\
\hline 5 & & $102(4.2)$ \\
\hline \multicolumn{3}{|l|}{ FEV $1 \%$ pred } \\
\hline Pre-bronchodilator & $2279(93.36)$ & $38.8 \pm 9.8$ \\
\hline Post-bronchodilator & $1510(61.86)$ & $42.2 \pm 11.2$ \\
\hline \multicolumn{3}{|l|}{ FVC $\%$ pred } \\
\hline Pre-bronchodilator & 2195 (89.92) & $66.2 \pm 17.5$ \\
\hline Post-bronchodilator & $1458(59.73)$ & $69.8 \pm 18.2$ \\
\hline \multicolumn{3}{|l|}{$\mathrm{FEV}_{1} / \mathrm{FVC} \%$} \\
\hline Pre-bronchodilator & $2077(85.09)$ & $47.1 \pm 10.4$ \\
\hline Post-bronchodilator & $1398(57.27)$ & $48.3 \pm 11.4$ \\
\hline Reversibility $^{+}$ & $1310(53.67)$ & \\
\hline$\%$ & & $9.07 \pm 14.9$ \\
\hline Yes & & 199 (15.2) \\
\hline Type of current COPD & $2431(99.59)$ & \\
\hline \multicolumn{3}{|l|}{ maintenance treatment } \\
\hline Only inhaled SABD ${ }^{\S}$ & & $50(2.1)$ \\
\hline Only one inhaled LABD ${ }^{f}$ & & $272(11.2)$ \\
\hline Only a combination of $L_{A B D^{\# \#}}^{\#}$ & & $94(3.9)$ \\
\hline Only inhaled glucocorticosteroids" & & $39(1.6)$ \\
\hline LABD plus inhaled glucocorticosteroids ${ }^{++}$ & & $1634(67.2)$ \\
\hline
\end{tabular}

Data are presented as $n$ (\%) or mean \pm SD. COPD: chronic obstructive pulmonary disease; MRC: Medical Research Council; FEV1: forced expiratory volume in $1 \mathrm{~s}$; \% pred: \% predicted; FVC: forced vital capacity; SABD: shortacting bronchodilator; LABD: long-acting bronchodilator. ${ }^{\#}: \mathrm{n}$ (\%) of the total population of 2,$441 ; "$ : MRC dyspnoea grade 1 , "I only get breathless with strenuous exercise"; grade 2, "I get short of breath when hurrying on the level or up a slight hill"; grade 3, "I walk slower than people of the same age on the level because of breathlessness or have to stop for breath when walking at my own pace on the level"; grade 4, "I stop for breath after walking $100 \mathrm{~m}$ or after a few minutes on the level"; grade 5, "I am too breathless to leave the house"; + : reversibility was calculated for patients with available pre- and postbronchodilator FEV1 data, and "yes" is defined as a post-bronchodilator increase in FEV1 of $\geqslant 200 \mathrm{~mL}$ and $\geqslant 12 \%$; ${ }^{\text {s }}$ : patients receiving only an inhaled short-acting $\beta_{2}$-agonist (SABA) or an inhaled short-acting anticholinergic, or a fixed or free combination of inhaled SABA plus anticholinergic; ${ }^{f}$ : patients receiving only one $\angle A B D$ (inhaled long-acting $\beta_{2}$-agonist (LABA), or oral LABA, or long-acting muscarinic antagonist (LAMA)) $\pm \mathrm{SABD} \pm$ methylxanthines; \#\#: patients receiving a combination of $\angle A B D(L A B A$ or $\angle A M A) \pm S A B D \pm$ methylxanthines; "थ: patients receiving only inhaled glucocorticosteroids \pm SABD; ${ }^{+}$: patients receiving inhaled glucocorticosteroids plus $L A B A$ (inhaled or oral) and/or LAMA \pm $\mathrm{SABD} \pm$ methylxanthines. 


\begin{tabular}{lc}
\hline TABLE 2 Patient comorbidities and current concomitant \\
medication \\
Comorbidity \\
Hypertension \\
Ischaemic heart disease & $1044(42.8)$ \\
Diabetes & $349(14.3)$ \\
Osteoporosis & $332(13.6)$ \\
Left heart failure & $266(10.9)$ \\
Depression (current) & $228(9.3)$ \\
Metabolic syndrome & $209(8.6)$ \\
Sleep apnoea & $208(8.5)$ \\
Any other condition that significantly affects mobility & $189(7.7)$ \\
Cancer (other than lung cancer) & $177(7.3)$ \\
Alcoholism & $150(6.1)$ \\
Concomitant medication & $120(4.9)$ \\
Diuretics & \\
Angiotensin-converting enzyme inhibitors & $620(25.4)$ \\
Statins & $521(21.3)$ \\
Angiotensin receptor blockers & $465(19.0)$ \\
Antidiabetics & $308(12.6)$ \\
Beta-blockers & \\
Antidepressants & $249(10.2)$ \\
Anxiolytics & $242(9.9)$ \\
Hypnotics & $179(7.3)$ \\
\hline & $150(6.1)$ \\
\hline
\end{tabular}

Data are presented as $n(\%)$ of the total population of 2,441. * : e.g. previous stroke or amputation; ${ }^{\bullet}$ : past or current; ${ }^{+}$: systemic and eye drops.

wheezing, cough and phlegm. A similar pattern was observed in the patients' perception of daily respiratory symptom variations (fig. 3). Breathlessness was the symptom that the largest group of patients reported as being variable, over the course of both a week and a single day.

All symptoms were most problematic firstly upon waking in the morning followed by "later in the morning" (except wheezing; fig. 4). The percentage of patients who reported troublesome symptoms upon waking and/or later in the morning was subsequently calculated: breathlessness, $45.4 \%$ $(n=804)$; cough, $60.1 \% \quad(n=861)$; phlegm, $70.9 \% \quad(n=1,100)$; chest tightness, $45.4 \%(n=313)$; and wheezing, $43.4 \%(n=442)$. These results show that, in general, symptomatic COPD is most troublesome in the morning.

\section{Factors associated with variability of breathlessness}

Multivariate analyses were performed to identify factors associated with breathlessness variability. Table 3 shows the odds ratios and the statistical significance for each of the factors identified. Factors associated with increased variation of breathlessness during the day were: younger age; high severity of breathlessness during the previous 7 days; and consultation with a GP rather than a specialist. Similar factors were associated with variation in breathlessness during the week. In addition, patients who had experienced two or more exacerbations in the previous 12 months were more likely to experience variability of breathlessness over the course of a week. Treatment with one long-acting bronchodilator, and left heart failure were associated with less variability during the day and the week, respectively. Sex, body mass index, current

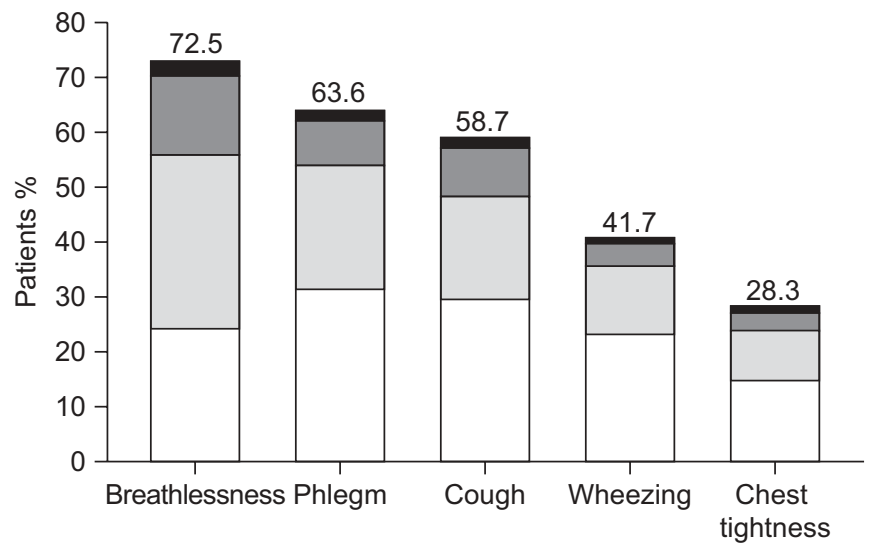

FIGURE 2. Patients were asked if they had experienced any symptoms (breathlessness $(n=1,769)$, phlegm $(n=1,552)$, cough $(n=1,433)$, wheezing $(n=1,018)$ or chest tightness $(n=690))$ in the 7 days prior to the telephone interview. Multiple answers were possible. $\square$ : a little; $\square$ : moderately; $\mathbf{\square}$ : very; extremely.

smoking, time since COPD diagnosis, MRC dyspnoea score, pre-bronchodilator \% predicted FEV1 and reversibility were not associated with breathlessness variability over the course of either a day or a week.

\section{Effect of symptoms on normal activities}

The morning activities that patients felt were most affected by their COPD symptoms were washing $(41.0 \%)$, dressing $(40.7 \%)$, drying $(36.2 \%)$ and getting out of bed $(35.4 \%)$. In total, $9.5 \%$ of the patient population $(n=231)$ reported that they required assistance to perform their normal morning activities and, of these patients, $67.5 \%(n=156)$ felt worried they were a burden to other people as a result. The daily activities that patients felt were most affected by COPD symptoms were going up and down stairs $(82.5 \%)$, doing heavy household chores $(56.9 \%)$, going shopping $(43.1 \%)$ and doing sport or hobbies $(35.9 \%)$. Impact on activities was more pronounced in patients reporting variability of breathlessness over the week $(p \leqslant 0.02$ for morning activities and $p \leqslant 0.001$ for other daily activities) or over the day ( $p \leqslant 0.001$ for morning activities). The impact on activities was also increased in those patients who reported the greatest variability of breathlessness ("a lot") compared with those with minimal variation ("a little") (supplementary tables 1, 2 and 3). Of the total cohort, 13.1\% $(n=319)$ stated that they required assistance to carry out their normal daily activities, and among these patients, $66.5 \%$ $(n=212)$ believed they imposed a burden upon other people because of this.

Overall, $9.4 \%$ of respondents were unable to exercise outside, but a third (30.9\%) reported walking outdoors for $>1 \mathrm{~h}$ per day during the previous 7 days, with $32.9 \%$ walking for 30-60 min per day and $24.7 \%$ managing up to $30 \mathrm{~min}$ per day.

A quarter of the total study population ( $n=646 ; 26.5 \%)$ reported that their symptoms had affected sleep quality. Specific aspects of sleep disturbance were identified as follows: frequent waking during the night $(n=516 ; 21.1 \%)$; difficulty falling asleep $(\mathrm{n}=461 ; 18.9 \%)$; and early waking in the morning $(n=407 ; 16.7 \%)$. Furthermore, night time was identified as one 


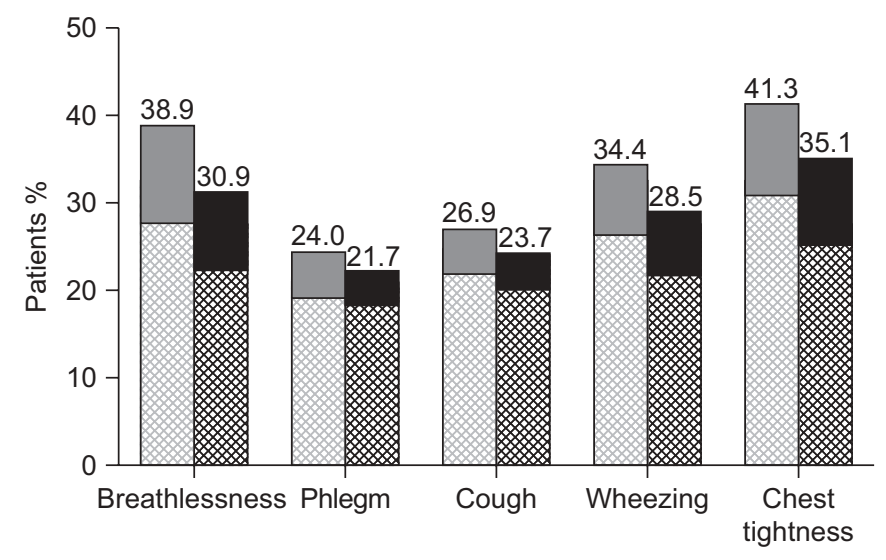

FIGURE 3. Patients who reported respiratory symptoms during the week prior to the telephone interview were asked if they experienced daily (black shading) and weekly (grey shading) variations in the severity of the symptom (breathlessness: $n=1,769$; phlegm: $n=1,552$; cough: $n=1,433$; wheezing: $n=1,018$; or chest tightness: $n=690$ ). Data shown are percentages of patients who answered "Yes, it varied a little" (hatched bars) or "Yes, it varied a lot" (solid bars).

of the most troublesome periods of the $24 \mathrm{~h}$ for a percentage of patients reporting breathlessness $(10.6 \%)$, chest tightness $(16.7 \%)$, cough $(17.3 \%)$, phlegm $(11.8 \%)$ or wheeze $(25.1 \%)$, respectively.

\section{Therapeutic behaviour and treatment compliance}

Maintenance therapy was used by $92.7 \%$ of the patients surveyed and $59.6 \%$ reported using a rescue inhaler. Among patients taking any medication ( $n=2,333 ; 96.7 \%)$, over half of them $(51.3 \%)$ reported that they did not alter their treatment despite symptoms worsening during the day; only $35.7 \%$ reported an increased use of their rescue inhaler. Similarly, $38.7 \%$ of patients declared that their use of medication remained unchanged when chest symptoms worsened over several days (fig. 5). Of those patients who did modify their treatment in response to worsening symptoms over several days, $28.3 \%$ reported an increase in rescue inhaler use, and $29.3 \%$ followed advice given by their physicians. Approximately 19\% $(n=457)$ of patients reported that they sometimes forget to take their daily medications and, among them, 36.5\% declared their symptoms worsen as a result.

There were also significant differences regarding therapeutic behaviour in response to worsening of symptoms over the day $(\mathrm{p}<0.001)$, depending on the degree of breathlessness variability reported by the patient ("no" variability, "a little" or "a lot"). However, no significant differences were observed according to weekly variability of symptoms. Patients reporting variability tended to be more likely to adapt their treatment in response to worsening symptoms (supplementary tables 4 and 5).

\section{Country or region data}

There were disparities regarding patient characteristics and COPD symptoms among the countries or regions. Daily and/ or weekly variability of one or more symptoms was perceived by $56.3-80.6 \%$ of patients $(\mathrm{p}<0.001)$. Differences were also observed for variability of breathlessness throughout the day $(p<0.001)$ and throughout the week $(p<0.001)$. The period of the day when breathlessness was the most troublesome varied but, for all countries or regions, "upon waking" was the most problematic time of the day. Rescue inhaler use was also significantly different between countries $(p<0.001)$ (supplementary tables 6-12).

\section{DISCUSSION}

The primary objective of the study was to examine patients' perceptions of daily, weekly and seasonal variability of respiratory symptoms, outside of exacerbation periods. Symptom variability was a frequent feature of the disease, reported by nearly two-thirds of patients with symptomatic COPD. For each individual symptom assessed, a significant number of patients (from $\sim 20 \%$ up to $40 \%$ ) reported that their symptoms varied over the course of the day and over the course of the week. Impact on activities was more pronounced in those patients reporting variability. Patients identified breathlessness in the morning as the most troublesome feature of the condition. This observation, using more robust methodologies, confirms other recent work in which the authors reported that patients perceived the morning as being the worst time of day for experiencing COPD symptoms and that shortness of breath was considered by the patients to be the most troublesome morning symptom and most likely to affect their quality of life (QoL) [22]. Following this observation, a patient questionnaire has been developed to assess morning symptoms in patients with COPD [23].

A recently validated short test enables clinicians to better assess the severity and impact of COPD [24]. Our findings show that specific attention should also be paid to variability of symptoms.

The association of troublesome morning symptoms and the impact they have on normal morning activities fits well with observations of circadian variations in lung function, such as FEV1, FVC and peak expiratory flow [7, 8, 25]. Another explanation could be that morning is the most active period of the day for severely impaired patients.

Identifying factors associated with variability of breathlessness also produced some interesting results. This symptom is the most prevalent in COPD, imposing a significant burden on QoL and the ability of patients to perform normal activities. The clarification of factors that either increase or decrease the variability of breathlessness is, therefore, important for the effective management of patients. The observation that patients recruited by a GP are more likely to experience variability of breathlessness than patients recruited by a specialist is unexpected and warrants further investigation. Although prescribed COPD medication was similar among patients recruited by a GP or a specialist (data not shown), one possible explanation is that GPs and specialists recruit different subgroups of COPD patients: for example, GPs might have included some patients with "asthmatic features". However, the variability was not correlated with reversibility of airflow obstruction after use of bronchodilators. Another explanation could be that patients with variability of breathlessness are more likely to seek medical assistance and access primarily their GP.

Increased variability of breathlessness during the day and the week was associated with severity of breathlessness, suggesting this feature may also reflect the severity of the respiratory impairment and its impact on exercise limitation. This may 

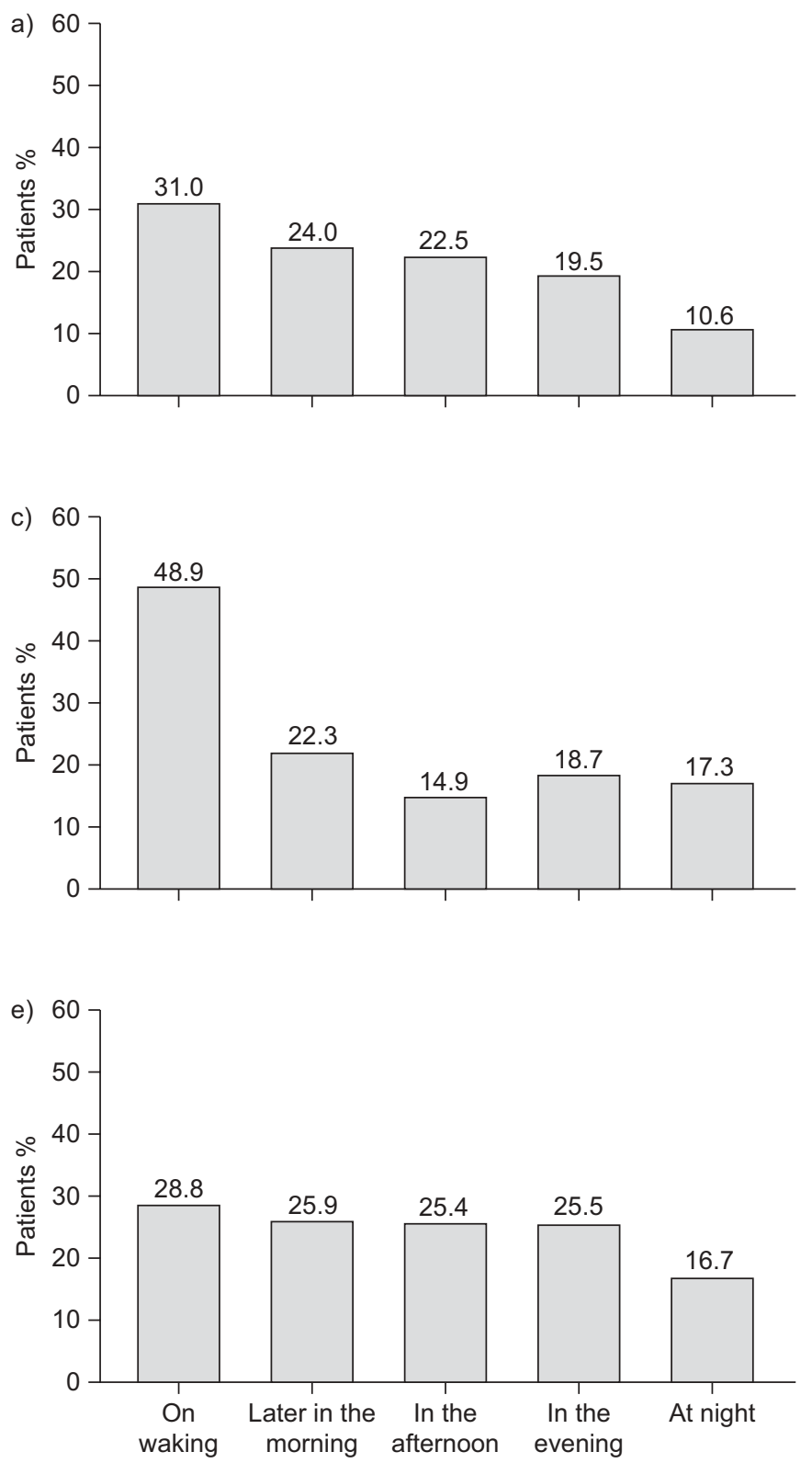

also explain why younger patients, who are generally more active, were more likely to report variability of breathlessness. The link between variability and level of physical activity, which significantly impacts health-related QoL [26], would need further investigation.

An interesting finding was that perceived variability of breathlessness during the week was associated with frequent exacerbations (two or more) in the previous year. This would appear to be consistent with a recent questionnaire-based study assessing patients' $(n=125)$ perspective of COPD exacerbations. The analysis identified breathlessness as one of the key attributes of exacerbations [5] and that descriptions of breathlessness were the most commonly patient-recollected warning of an exacerbation [15].

Another finding of this study was the group's response as to how they modified their use of COPD medications during b)

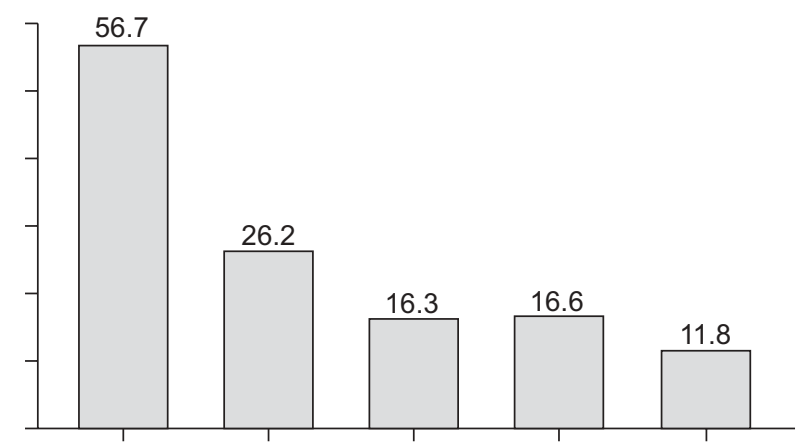

d)

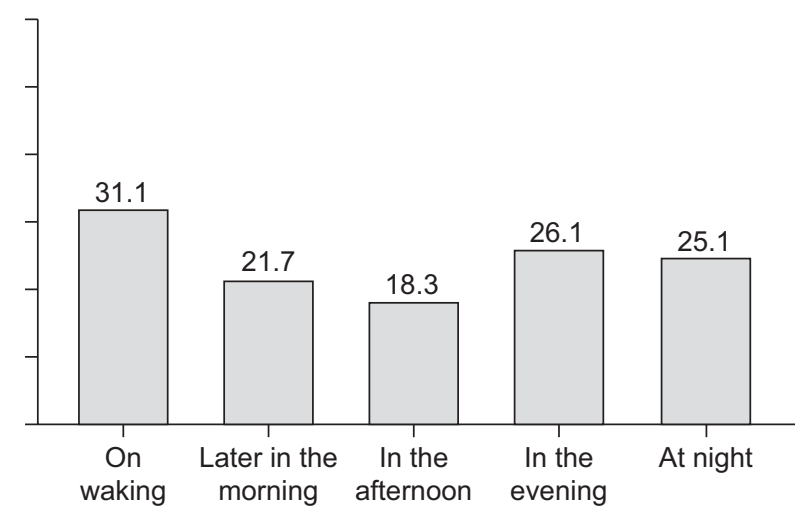

FIGURE 4. Patients who had reported experiencing symptoms in the previous 7 days were asked during what times of the day the symptoms were most troublesome. a) Breathlessness, $n=1,769$; b) phlegm, $n=1,551$; c) cough, $n=1,433$; d) wheezing, $n=1,018$; and e) chest tightness, $n=690$.

periods of symptom variability. In contrast to the recommendations in the Global Initiative for Chronic Obstructive Lung Disease guidelines, the use of rescue inhaler medication was rather limited [1]. The observation that approximately half of all patients continued to use their medications in the same way when symptoms were more troublesome than usual suggests that patient education regarding the use of different COPD medications could be improved, and that the impact of symptom variability might be reduced as a result. A recent study comparing the effects of different combinations of inhaled glucocorticosteroids and long-acting bronchodilators, when taken early in the morning, supports this hypothesis, suggesting that patients are taking medications too late in the day to benefit fully from their potential effects [27].

This study shows disparities in the patients' characteristics between region and countries. For example, there were more female patients, more leaner patients and more current 
TABLE 3 Determination of factors that may influence breathlessness variation during the day and week ${ }^{\#}$

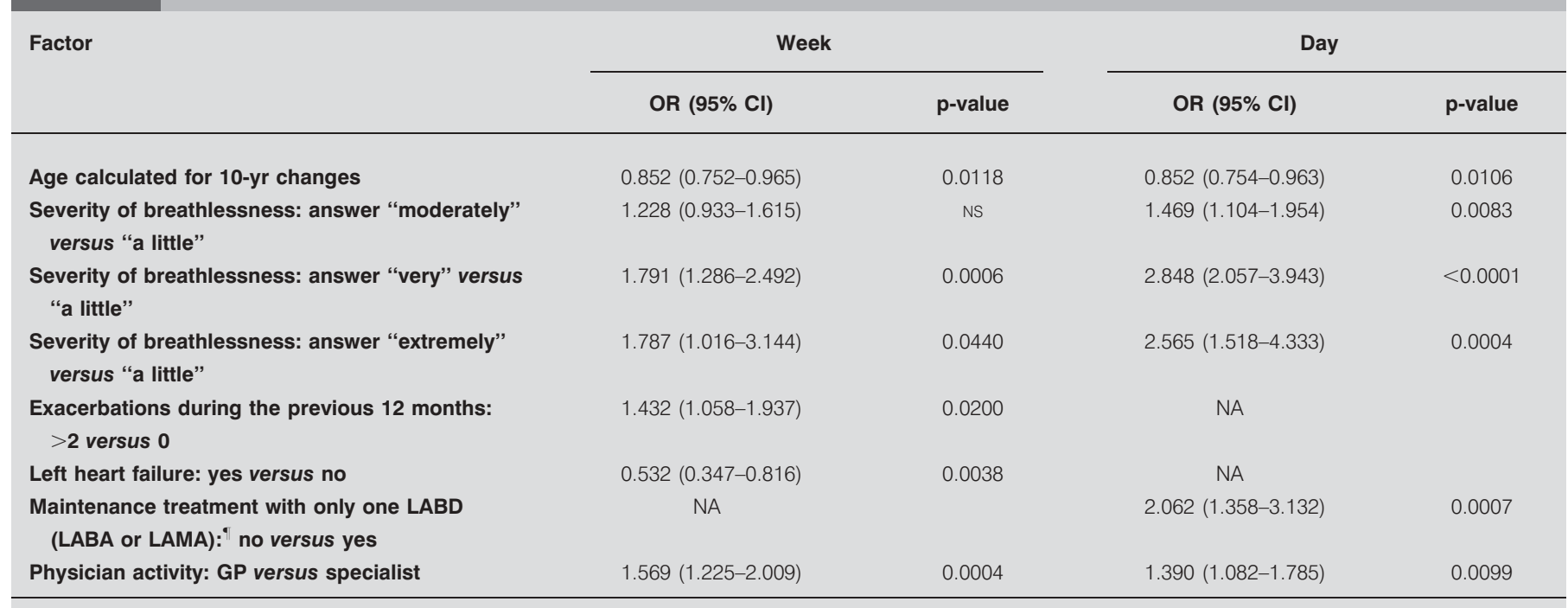

NS: not significant; NA: not significant in the univariate analysis and therefore not included in the multivariate analysis; LABD: long-acting bronchodilator; LABA: longacting $\beta_{2}$-agonist; LAMA: Iong-acting muscarinic antagonist; GP: general practitioner. ${ }^{*}$ : each factor listed was considered significant in the univariate analysis ( $\left.p=0.20\right)$ and thus eligible for inclusion in the logistic regression model. Factors were then selected using a backward, stepwise procedure with a significance level of $p=0.05$ for entering or removing variables. Patients who reported breathlessness during the past 7 days $(n=1,769)$ and who answered the specific question regarding weekly $(n=1,733)$ or daily variability $(n=1,668)$ were included in the univariate analysis. ": "yes" indicates patients taking only one LABD \pm short-acting bronchodilator \pm methylxanthines; "no" indicates other maintenance treatment (see table 1).

smokers included in the Scandinavian region than in Spain. These disparities could, in part, explain the differences in perception of variability according to geographic area.

The primary limitation of this study is whether recruited patients were suffering from COPD only, or whether any reported variability was due to the inadvertent inclusion of patients with asthma. Indeed, patients with combined diseases (asthma and COPD) are not infrequent [28, 29]. To control for this possibility, patients with a history of asthma were specifically excluded from the cohort. Of interest, reversibility was not a factor associated with variability of breathlessness in this study. Another possible limitation is whether patients really were in a stable disease state. Previous research has shown that exacerbation profiles vary considerably between patients and that the patients themselves often have a poor understanding of what, exactly, the term exacerbation means [15]. Participants should not have experienced a severe
Therapeutic behaviour

the dose and/or frequency of

1) Vary the dose and/or frequency of
regular daily medication

2) Use more of emergency inhaler

3) Continue using the medicine in the same way

Don't know/don't want to answer

1) +2

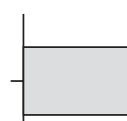

Symptoms worsen during the day
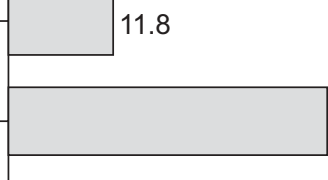

35.7

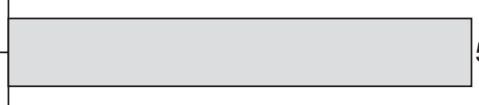

51.3

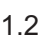

1.2

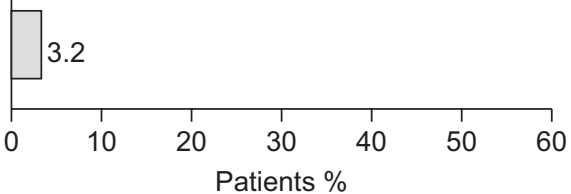

Symptoms worsen over several days

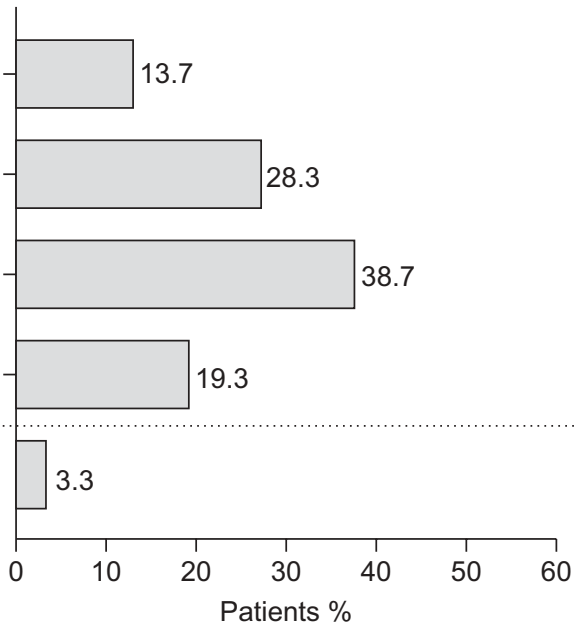

FIGURE 5. Patients were asked how, if at all, they modified the use of their COPD medications when experiencing symptom variability during the day $(n=2,333)$ or over the course of several days $(n=2,333)$. 
exacerbation, leading to therapeutic intervention, in the 3 months prior to the study. However, it is possible that less severe exacerbations were reported as symptom variability; follow-up studies have shown that some exacerbations may not be reported by patients [30]. The cross-sectional design of the study might be considered suboptimal for the assessment of the variability of disease symptoms. Nevertheless, this design allowed us to assess the perception of symptoms in a large sample of patients in a clinical setting. Patients were asked to recall symptoms and perception of variability, and not to complete diaries, as this observational study was replicating real-life clinical practice. The phone interview methodology was chosen because it allowed patients' perceptions to be collected, with no influence from their physician and/or a proxy.

This study has shown that in between exacerbation periods, patients with severely impaired lung function often perceive both daily and weekly variability of their COPD symptoms and that the morning is the most troublesome time of day. Variability of breathlessness was associated with more severe dyspnoea and increased exacerbation frequency. Only a minority of patients seem to adapt their treatment in response to worsening of symptoms.

The observations recorded during this study have important implications for patients with severe COPD and may lead to improvements in how their condition is managed both day-today and over the long term. Further prospective longitudinal studies investigating COPD symptom variability and associated mechanisms are needed to shed more light on the extent of symptom variability and the impact this has on patients' normal routines.

\section{SUPPORT STATEMENT}

This study was funded by AstraZeneca.

\section{CLINICAL TRIALS}

This study is registered at clinicaltrials.gov with identify number NCT00722267.

\section{STATEMENT OF INTEREST}

Statements of interest for R. Kessler, M.R. Partridge, M. Miravitlles, M. Cazzola, D. Leynaud and J. Ostinelli, and for the study itself, can be found at www.erj.ersjournals.com/site/misc/statements.xhtml

\section{ACKNOWLEDGEMENTS}

We thank all participating physicians and patients for their involvement in the present study (a full list of physicians is available in the supplementary material). We also thank B. Jepson, from Fishawack Communications Ltd, Knutsford, UK, who provided medical writing support funded by AstraZeneca. The operational conduct of the study including physician recruitment, patient interview, data collection and management was co-ordinated by Mapi Group, Lyon, France, a contract research organisation; the statistical analysis was performed by Sami Boussetta (Mapi Group).

\section{REFERENCES}

1 Rabe KF, Hurd S, Anzueto A, et al. Global strategy for the diagnosis, management, and prevention of chronic obstructive pulmonary disease: GOLD executive summary. Am J Respir Crit Care Med 2007; 176: 532-555.

2 Clark TJ. Diurnal rhythm of asthma. Chest 1987; 91: 137S-141S.
3 Hetzel MR, Clark TJ. Comparison of normal and asthmatic circadian rhythms in peak expiratory flow rate. Thorax 1980; 35: 732-738.

4 Turner-Warwick M. Epidemiology of nocturnal asthma. Am J Med 1988; 85: 6-8.

5 Haughney J, Partridge MR, Vogelmeier C, et al. Exacerbations of COPD: quantifying the patient's perspective using discrete choice modelling. Eur Respir J 2005; 26: 623-629.

6 Lewinsohn HC, Capel LH, Smart J. Changes in forced expiratory volumes throughout the day. Br Med J 1960; 1: 462-464.

7 Postma DS, Koeter GH, vd Mark TW, et al. The effects of oral slowrelease terbutaline on the circadian variation in spirometry and arterial blood gas levels in patients with chronic airflow obstruction. Chest 1985; 87: 653-657.

8 van Noord JA, Aumann JL, Janssens E, et al. Effects of tiotropium with and without formoterol on airflow obstruction and resting hyperinflation in patients with COPD. Chest 2006; 129: 509-517.

9 Barnett M. Chronic obstructive pulmonary disease: a phenomenological study of patients' experiences. J Clin Nurs 2005; 14: 805-812.

10 Barr RG, Celli BR, Martinez FJ, et al. Physician and patient perceptions in COPD: the COPD Resource Network Needs Assessment Survey. Am J Med 2005; 118: 1415.

11 Engstrom CP, Persson LO, Larsson S, et al. Health-related quality of life in COPD: why both disease-specific and generic measures should be used. Eur Respir J 2001; 18: 69-76.

12 Ferrer M, Alonso J, Morera J, et al. Chronic obstructive pulmonary disease stage and health-related quality of life. The Quality of Life of Chronic Obstructive Pulmonary Disease Study Group. Ann Intern Med 1997; 127: 1072-1079.

13 Jones PW. Quality of life measurement for patients with diseases of the airways. Thorax 1991; 46: 676-682.

14 Rutten-van Molken MP, Oostenbrink JB, Tashkin DP, et al. Does quality of life of COPD patients as measured by the generic EuroQol five-dimension questionnaire differentiate between COPD severity stages? Chest 2006; 130: 1117-1128.

15 Kessler R, Stahl E, Vogelmeier C, et al. Patient understanding, detection, and experience of COPD exacerbations: an observational, interview-based study. Chest 2006; 130: 133-142.

16 Miravitlles M, Anzueto A, Legnani D, et al. Patient's perception of exacerbations of COPD—the PERCEIVE study. Respir Med 2007; 101: 453-460.

17 Miravitlles M, Ferrer M, Pont A, et al. Effect of exacerbations on quality of life in patients with chronic obstructive pulmonary disease: a 2 year follow up study. Thorax 2004; 59: 387-395.

$18 \mathrm{Ng}$ TP, Niti M, Tan WC, et al. Depressive symptoms and chronic obstructive pulmonary disease: effect on mortality, hospital readmission, symptom burden, functional status, and quality of life. Arch Intern Med 2007; 167: 60-67.

19 Katula JA, Rejeski WJ, Wickley KL, et al. Perceived difficulty, importance, and satisfaction with physical function in COPD patients. Health Qual Life Outcomes 2004; 2: 18.

20 Gilbert C, Martin ML, Hareendran A, et al. Capturing individual variation in the experience of symptoms reported by patients with COPD. Am J Respir Crit Care Med 2007; 175: A15-1004.

21 Willams SJ. Chronic Respiratory Illness. London, Routledge, 1993.

22 Partridge MR, Karlsson N, Small IR. Patient insight into the impact of chronic obstructive pulmonary disease in the morning: an internet survey. Curr Med Res Opin 2009; 25: 2043-2048.

23 Partridge MR, Miravitlles M, Stahl E, et al. Development and validation of the Capacity of Daily Living during the Morning questionnaire and the Global Chest Symptoms Questionnaire in COPD. Eur Respir J 2010; 36: 96-104.

24 Jones PW, Harding G, Berry P, et al. Development and first validation of the COPD Assessment Test. Eur Respir J 2009; 34: 648-654. 
25 Calverley PM, Lee A, Towse L, et al. Effect of tiotropium bromide on circadian variation in airflow limitation in chronic obstructive pulmonary disease. Thorax 2003; 58: 855-860.

26 Esteban C, Quintana JM, Aburto M, et al. Impact of changes in physical activity on health-related quality of life among patients with COPD. Eur Respir J 2010; 36: 292-300.

27 Partridge MR, Schuermann W, Beckman O, et al. Effect on lung function and morning activities of budesonide/formoterol versus salmeterol/fluticasone in patients with COPD. Ther Adv Respir Dis 2009; 3: 1-11.
28 Soriano JB, Davis KJ, Coleman B, et al. The proportional Venn diagram of obstructive lung disease: two approximations from the United States and the United Kingdom. Chest 2003; 124: 474-481.

29 Gibson PG, Simpson JL. The overlap syndrome of asthma and COPD: what are its features and how important is it? Thorax 2009; 64: 728-735.

30 Cazzola M, MacNee W, Martinez FJ, et al. Outcomes for COPD pharmacological trials: from lung function to biomarkers. Eur Respir J 2008; 31: 416-469. 